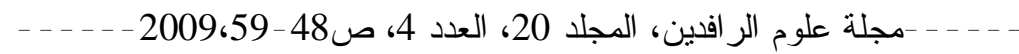

\section{دراسة الفعالية المناعية للمتمم في مصول الأصحاء خلال الصيام}

\author{
زياد ذنون الرسام \\ قسم علوم الحباة \\ كلبة العلوم \\ جامعة الموصل \\ (تاريخ الاستلام 15/ 7 / 2009 ؛ تاريخ القبول 28 /9 / 2009)

\section{الملخص}

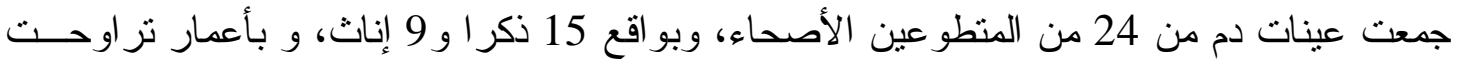

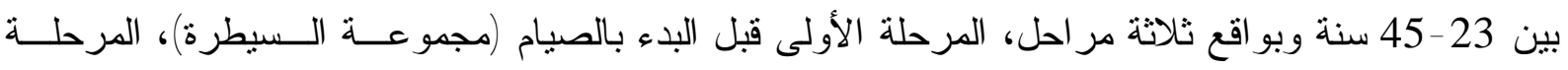

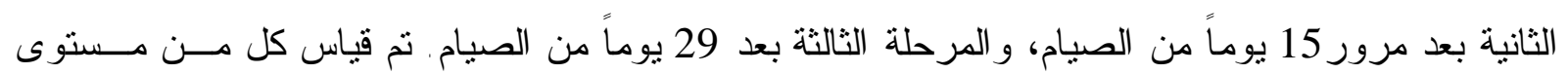

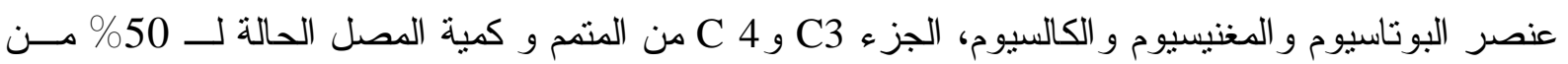

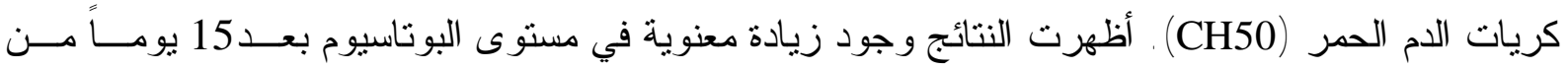

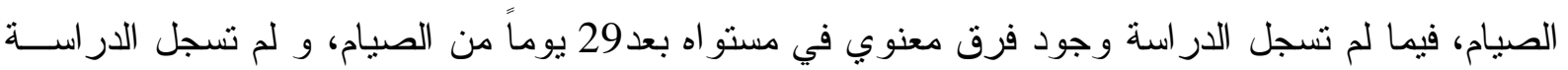

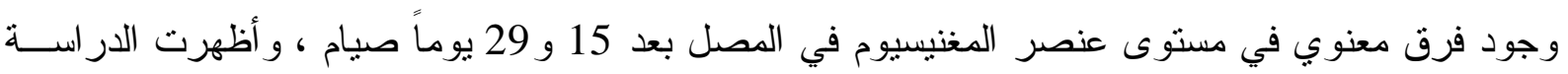

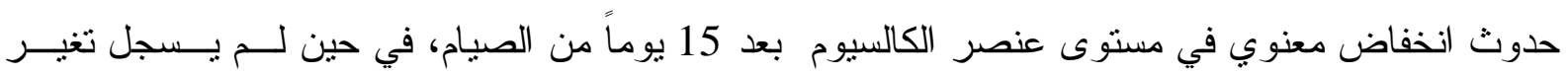

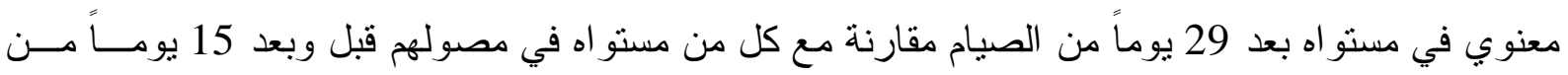

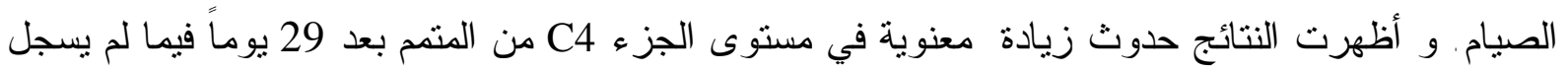
وجود فرق معنوي في مستوى الجزء C3 خلال الفتر ات الثلاثة، كما سجلت الدر اسة حدوث انخفاض معنوي

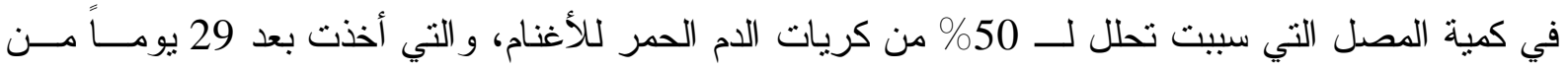

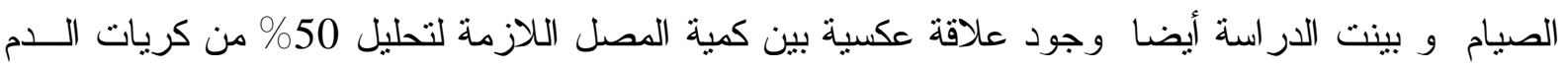

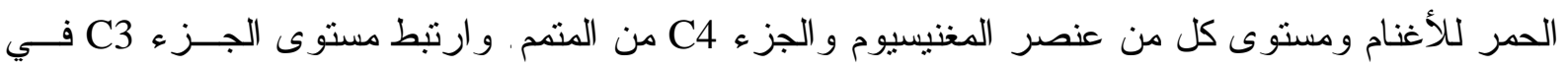

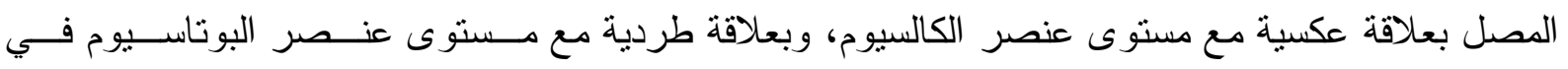
المصل، وارتبط مستوى عنصر الكالسيوم بعلاقة عكسية مع مستوى عنصر البوتاسيوم في المصل. الكلمات الدالة: الفعالية المناعية للمتم خلال صيام. 


$$
\text { زياد ذنون الرسام }
$$

\title{
Study the Immunological Activity of Complement in the Sera of Healthy Subjects During Fasting
}

\author{
Zeyad T. Al-Rassam \\ Department of Biology \\ College of Science \\ Mosul University \\ zeyadalrassam@yahoo.com
}

\begin{abstract}
Blood samples were collected from 24 healthy donors, 15 males and 9 females ranging in age from 23-45 years. Three blood samples were obtained from each subject, before fasting, 15 days and 29 days after fasting. Serum potassium, Magnesium, Calcium and complement $\mathrm{C} 3, \mathrm{C} 4$ and quantity of serum causing hemolysis for $50 \%$ of RBCs (CH50) were calculated.

The results showed a significant increase in the level of Potassium 15 days after fasting while no difference were recorded in its level after 29 days after fasting, and the results showed a significant decrease in serum Calcium level after 15 days, while no significant difference occurs after 29 days compared with its level before and after 15 days of fasting. The results showed a significant increase in the level of complement component C4 after 29 days of fasting while no significant difference were recorded in the level C3 complement component during the three experimental stages, the results also recorded a significant decrease in the amount of serum which causes hemolysis for 50\% of SRBC after 29 days of fasting, and the results also showed an inverse correlation between the amount of serum causing hemolysis for 50\% of SRBC and serum Magnesium level and complement $\mathrm{C} 4$ level, and an inverse correlation between $\mathrm{C} 3$ complement component and Calcium level while proportional correlation with Potassium serum level, and an inverse correlation with serum Potassium level.
\end{abstract}

Keywords: Complement Activity During Fasting.

$$
\begin{aligned}
& \text { المقدمة } \\
& \text { الصيام ليس الامتتاع عن الطعام و الثراب وملذات الحياة لساعات معدودة من النهار ، بل هو تغير فــي } \\
& \text { العادات السلوكية للإنسان (Akuchekian et al., 2004). و هو إستر اتيجية لها اثر ايجابي في تحسين تحمل }
\end{aligned}
$$

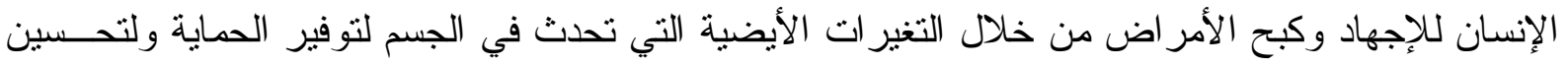

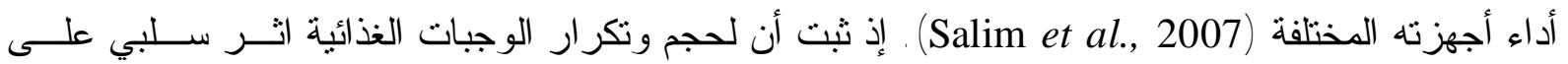

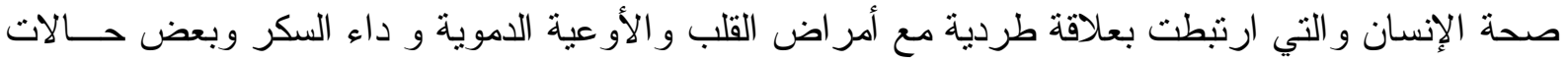

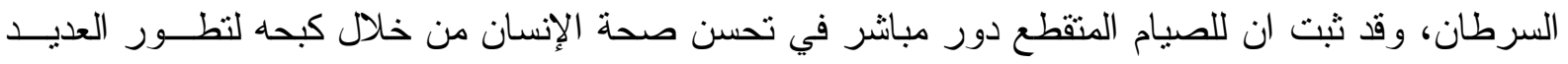

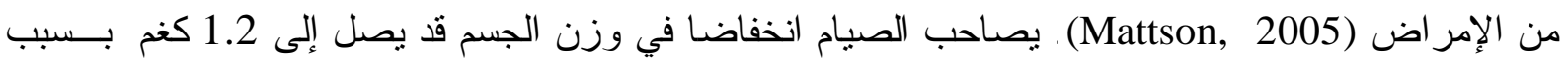


الجفاف وفقدان الماءو واستهلاك 5\% من الدهون المخزونة في الجسم كمصدر للطاقة وخصوصـا فـي الأيسام

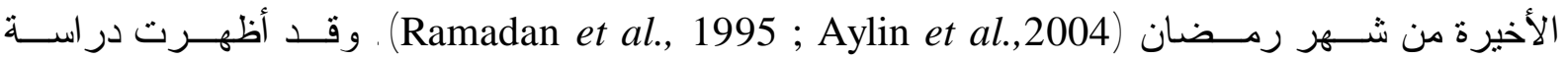
(Frost and Pirani, 1987)

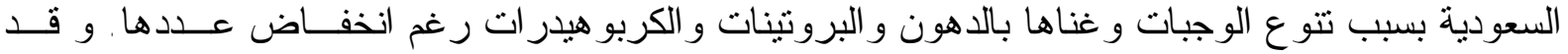
أثنتت در اسات (Kiziltan et al., 2005 ; Abolfazl et al., 2007) عدم وجود اثر سلبي للصيام على صحة

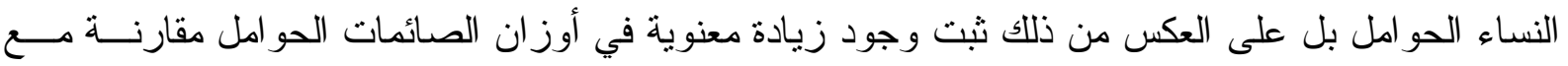
أوزان الحوامل غير الصائمات، وان أوزان الأطفال المولودين من أمهات صائمات خلال رمضان كانو أكثر

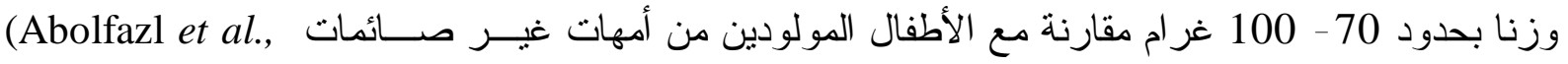

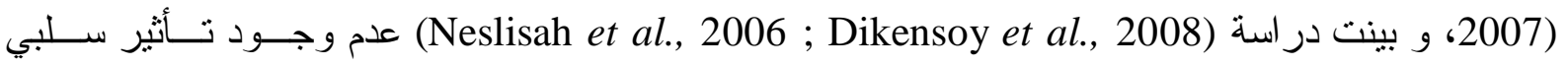

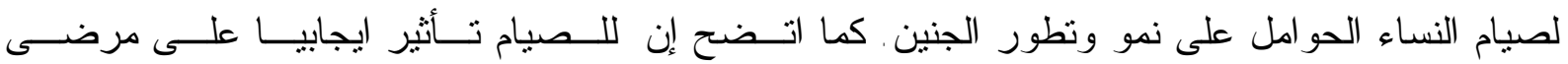

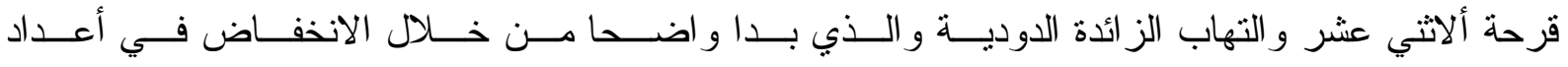

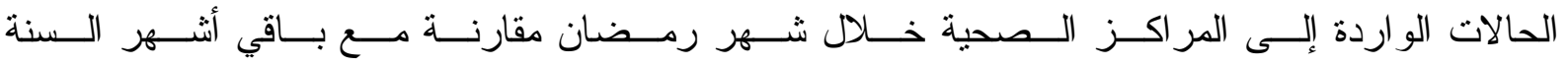
(Davoodabadi et al., 2005 ; Abolfazl et al., 2007 ; Tavakkoli et al., 2008)

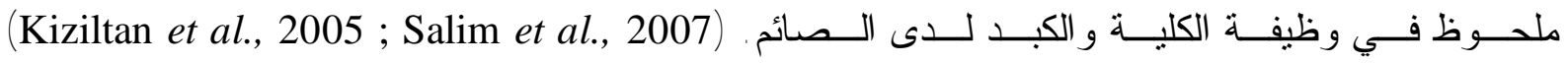

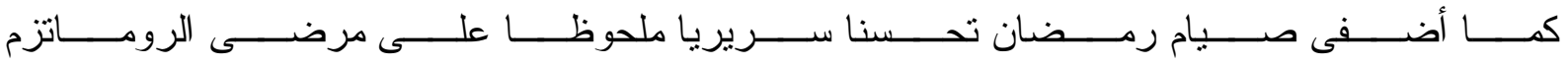
(Al-Dubeikil and Abdul-Lateef, 2003) و وبناء على ما تقدم يمكننا القول إن هذا النظام المنهجي للحياة

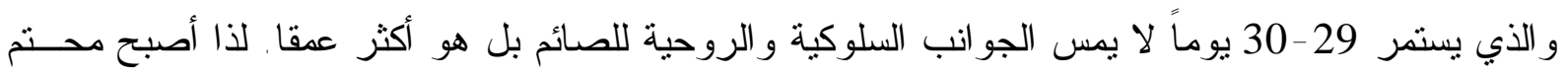

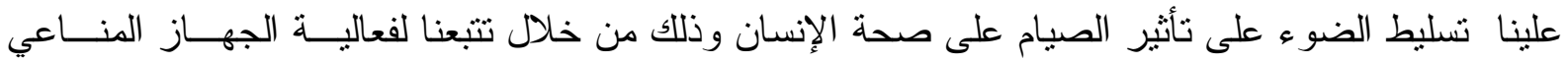
لجسم الصائم على مدار شهر رمضان.

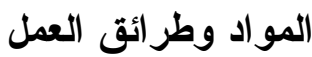

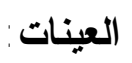

شملت الدر اسة 24 من المنطو عين الأصحاء من منتسبي قسم علوم الحياة / جامعة الموصـل وبعــ

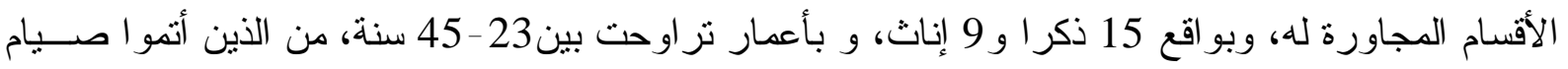

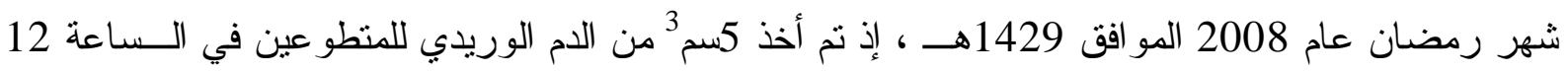

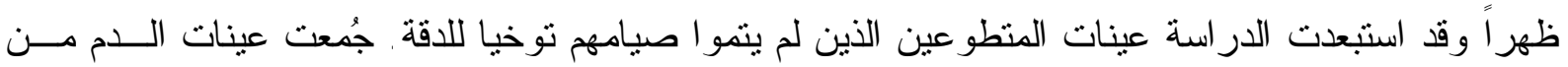

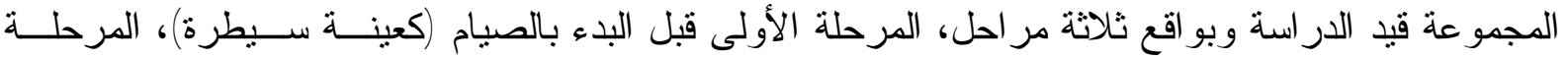

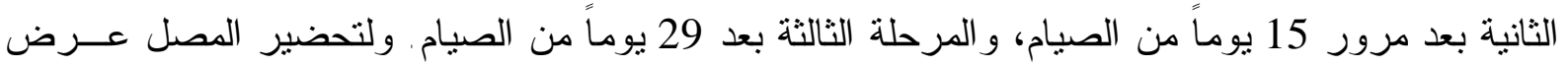


الدم للطرد المركزي بسرعة 3000 دورة في الدقيقة ولمدة 15 دقيقة وباستخدام جهاز الطرد المركزي من

نو ع REMI هندي المنشأ. فصل الهصل ووزع على أنابيب صغيرة وتم حفظها لحين إجر اء الاختبار ات.

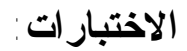

1 - نم قياس مستوى الجزء C3 للمتمم في مصول المتطوعين قبل البدء بالصيام، بعد مرور 15 يومــاً مـنـ

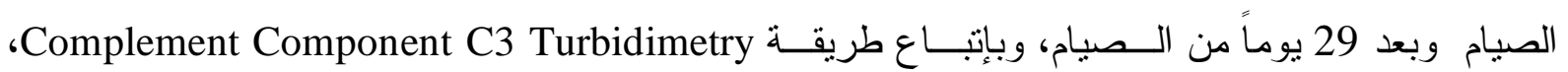
باستخدام عدة الفحص COD:31084 المجززة من قبل شركة Biosystems وحسب طريقة العمـلـ المرفقــة مع عدة الفحص. 2- تم قياس مستوى الجزء C4 للمتمم في مصول المتطو عين قبل البدء بالصيام، بعد 15 يوماً مــن الـصيام

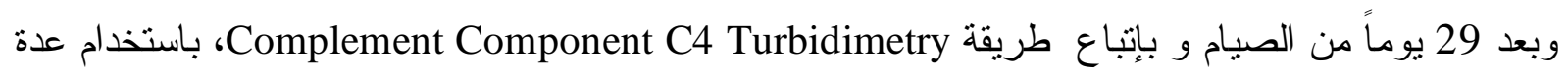

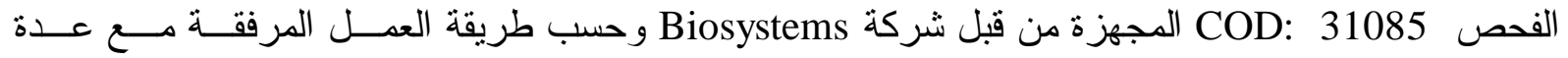
الفحص. 3- تم قياس فعالية المصل الحالة لـــ50\% من كريات الدم الحمر للأغنام في مصول المنطو عين ومقارنتــهـ في المر احل الثلاثة وحسب طريقة (Hudson and Hay , 1980).

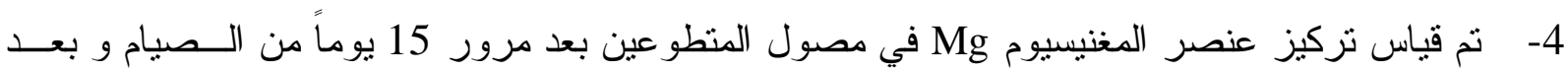

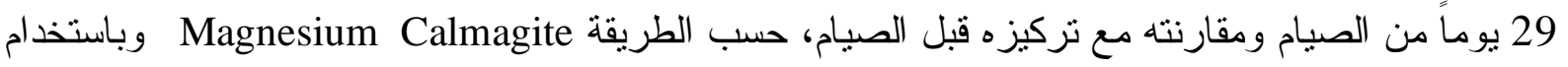
عدة الفحص COD:11512 المجزة من قبل شركة Biosystems وباتباع طريقة العمل المرفقــة مــع عـدة فئس الفحص.

5- ت تم قياس تركيز كل من عنصر البوناسيوم K و عنصر الكالسيوم Ca في مصول المنطو عين بعـد مــرور

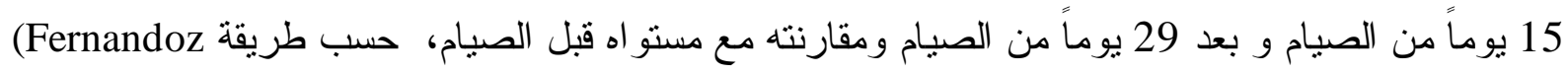
and Khan, 1971) وباستخدام جهاز امتصاص الطيف الذري Atomic Absorption المجهز من قبل شركة Pye Unicam ، الموجود في مختبر البحوث في قسم علوم الحياة / كلية العلوم/ جامعة الموصل.

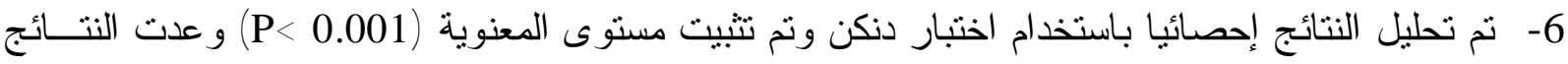
معنوية عند مستوى0.05

\section{النتائج و المناقثة}

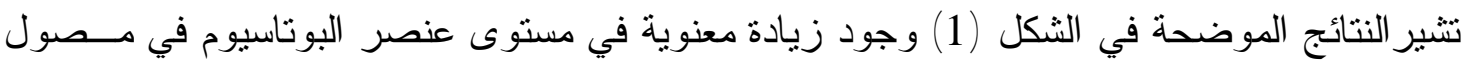

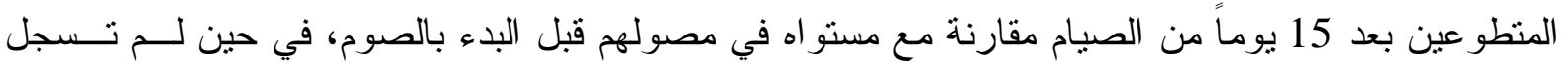

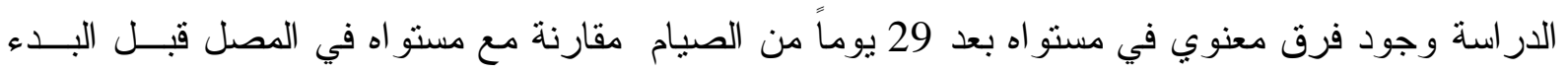

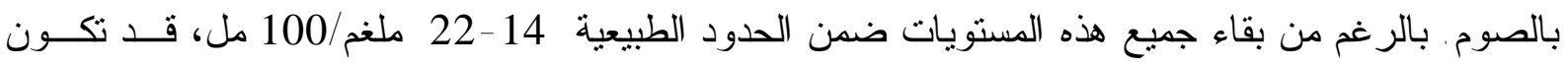

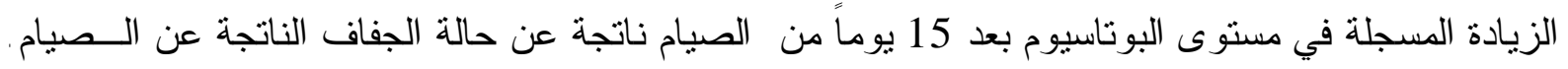




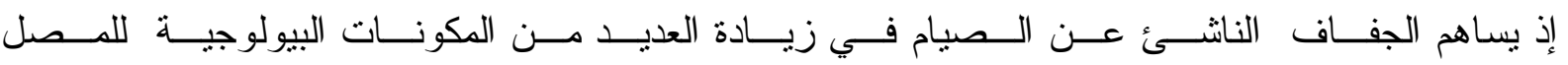
(Fedail et al., 1982 ; Ramadan et al., 1999 ; Leiper et al., 2003)

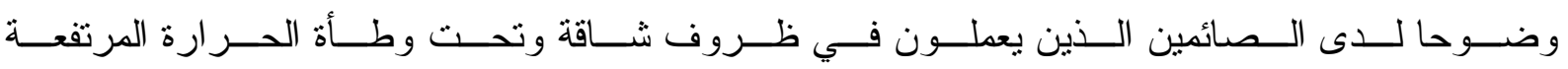

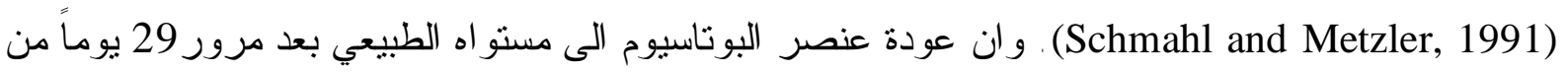
الصيام دليل على تكيف الجسم مع حالة الصوم (Sheah et al., 1990).

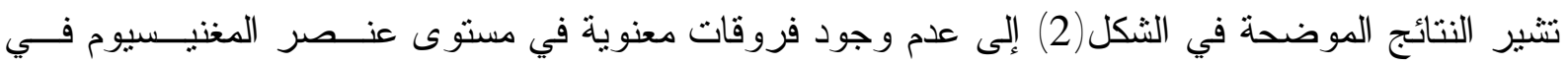

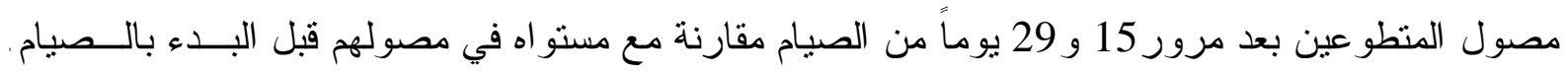

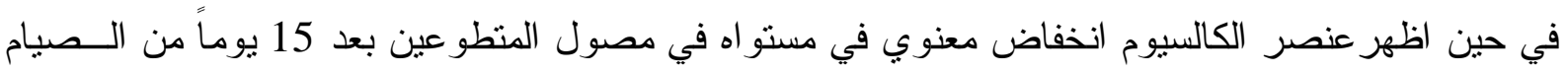

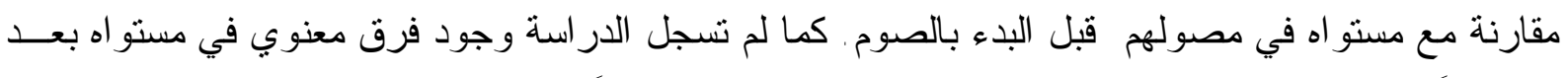

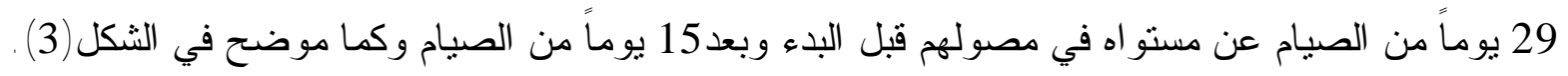

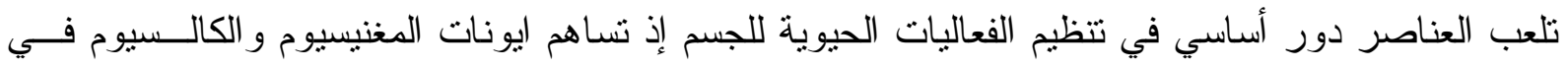

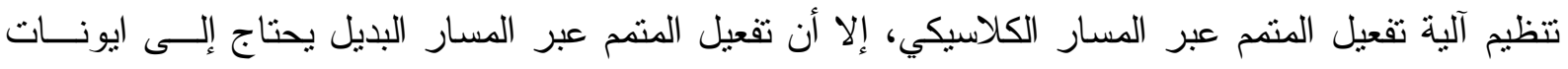

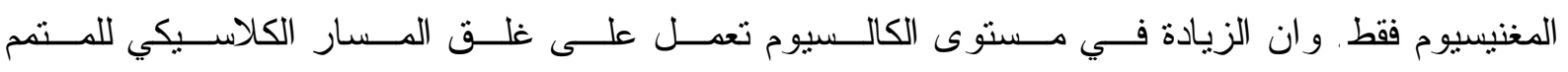

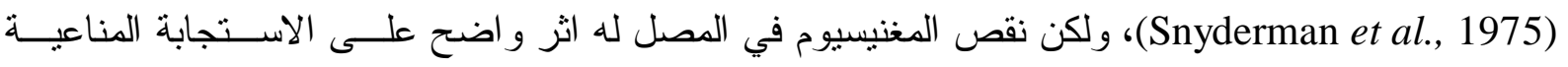
للجسم (Bussiere et al., 2003)، إذ أوضحت دراسة (Spacek et al., 2005) إن نكر ار الإصـابة بخميـرة Recurrent Vulva vaginal Candidosis (RVC) Candida

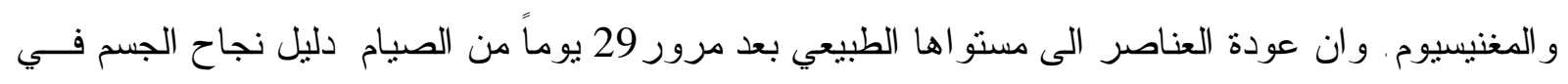

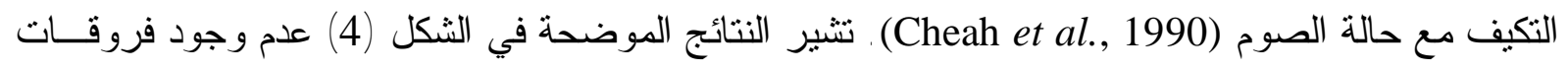

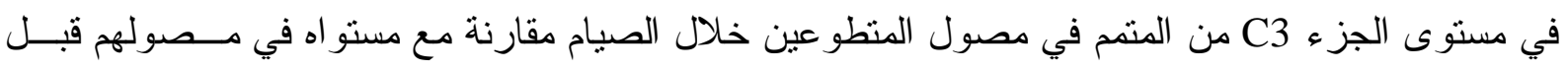

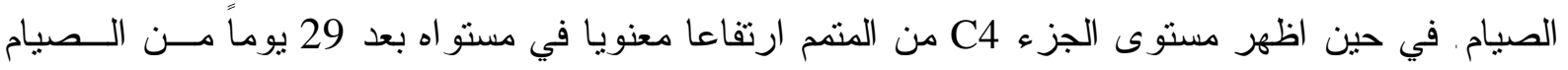

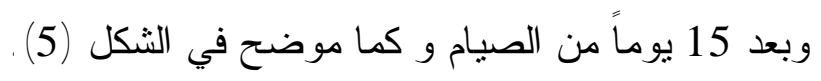

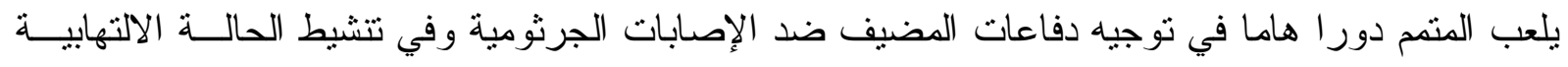

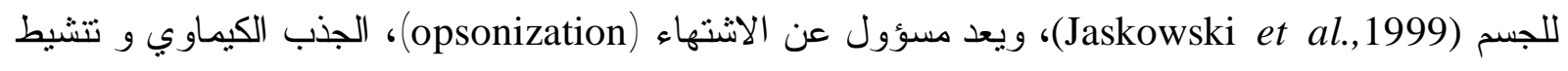

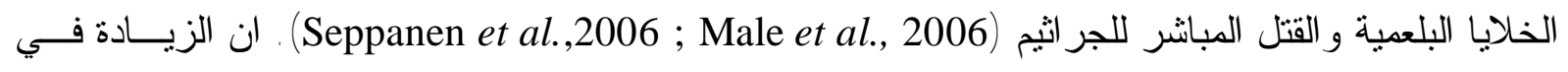

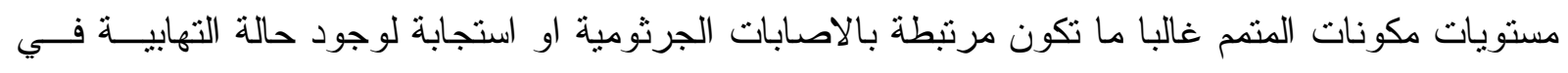

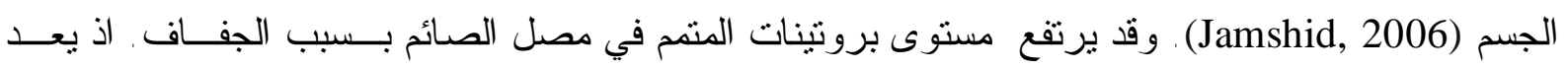

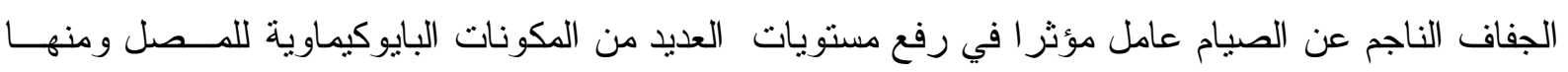
بروتينـات المـصل (Ramadan et al., 1995; Ramadan et al., 1999; Gutierrez et al., 2001) وخصوصا لدى الصائمين الذين يعملون في ظروف شاقة وحر ارة مرتفعة (Schmahl and Metzler,1991). 


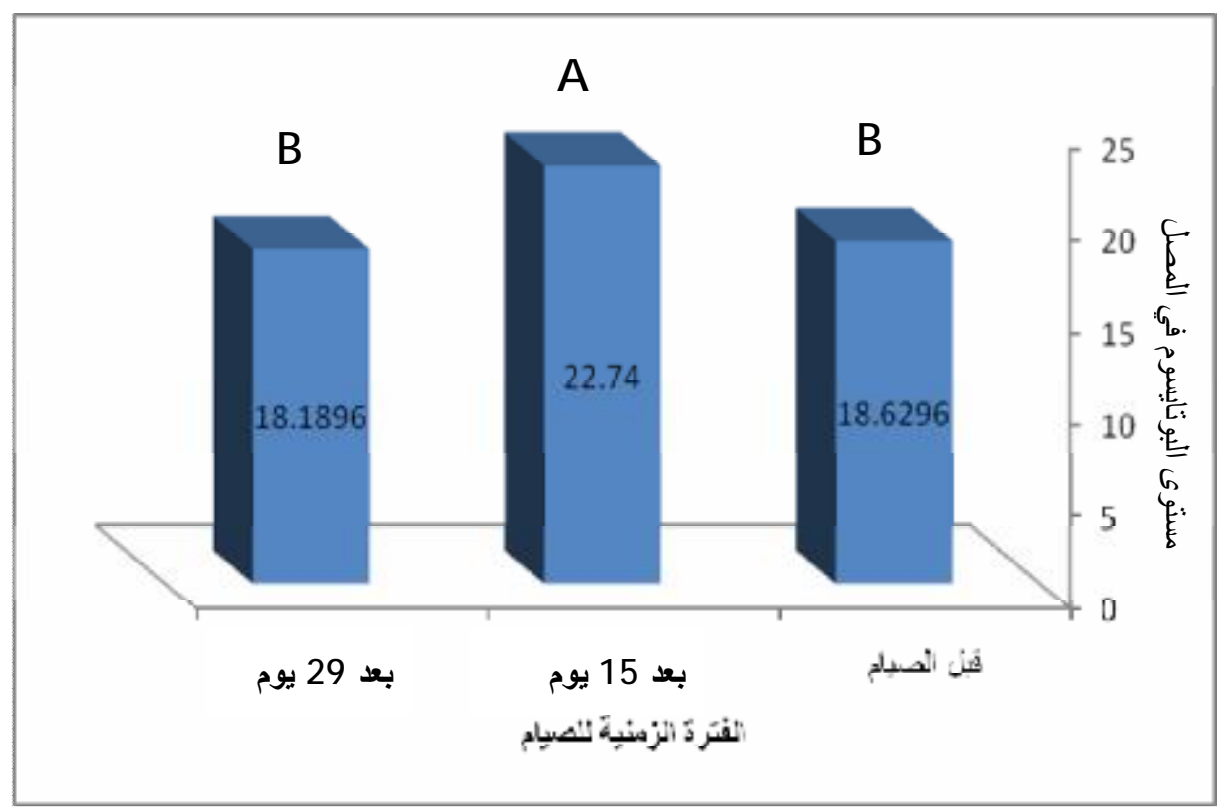

المعدلات ذات الحروف المختلفة تعني وجود فرق معنوي عند مستوى احتمالية 0.01 حسب اختبار دنكن

الثكل 1 : مستوى عنصر البوتاسيوم في مصول المتطو عين قبل و خلال صيام شهر رمضنان

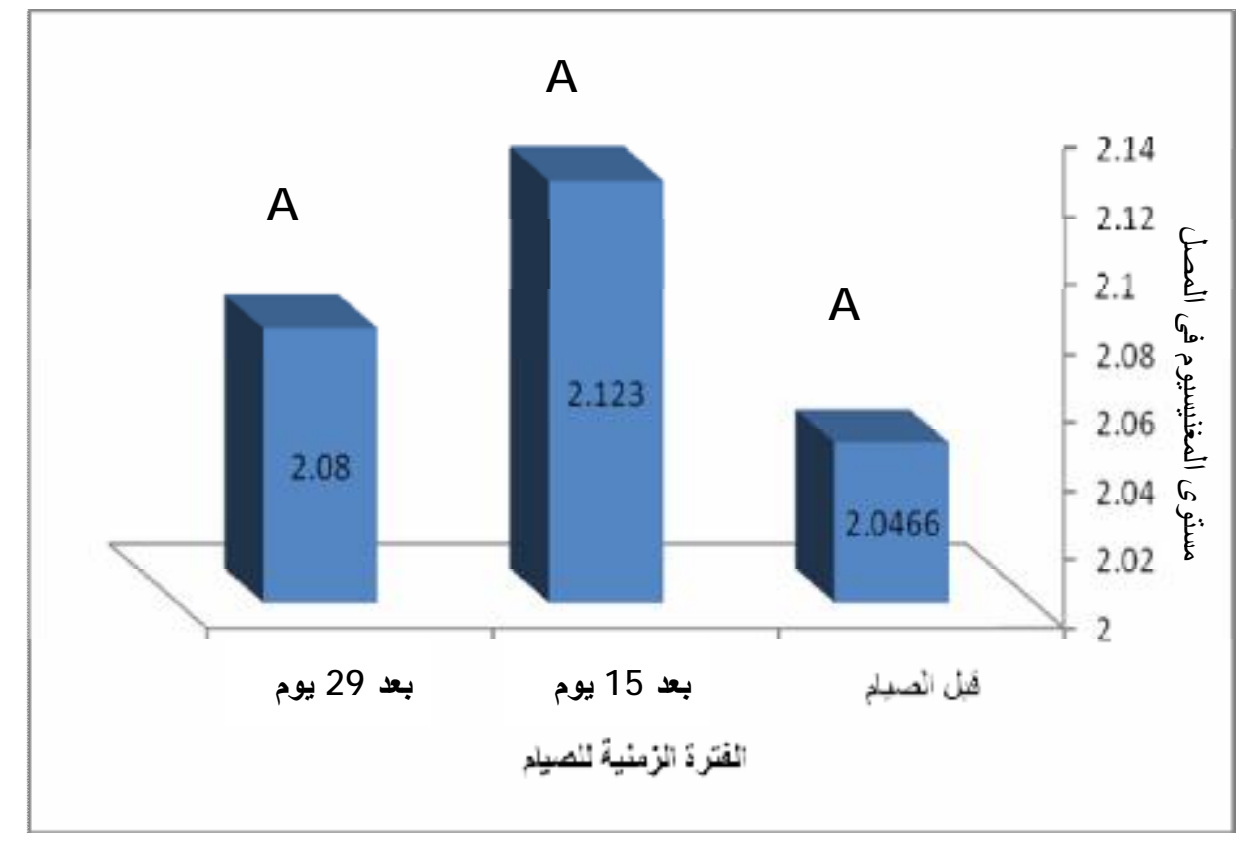

المعدلات ذات الحروف المختلفة تعني وجود فرق معنوي عند مستوى احتمالية 0.01 حسب اختبار دنكن

الشكل 2 : مستوى عنصر المغنيسيوم في مصول المتطو عين قبل و خلال صيام شهر رمضان 


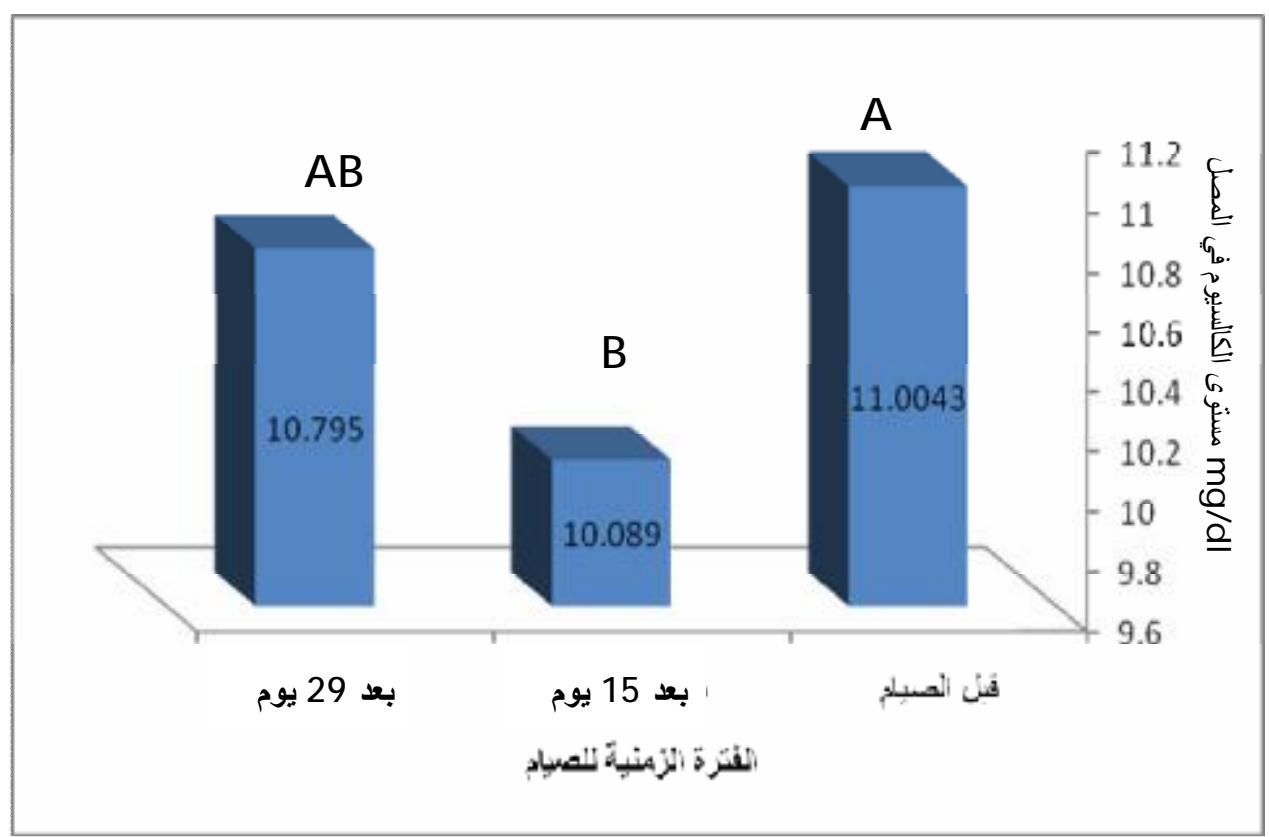

المعدلات ذات الحروف المختلفة تعني وجود فرق معنوي عند مستوى احتمالية 0.01 حسب اختبار دنكن

الثكل 3 : مستوى عنصر الكالسيوم في مصول المتطوعين قبل و خلال صبام شهر رمضان

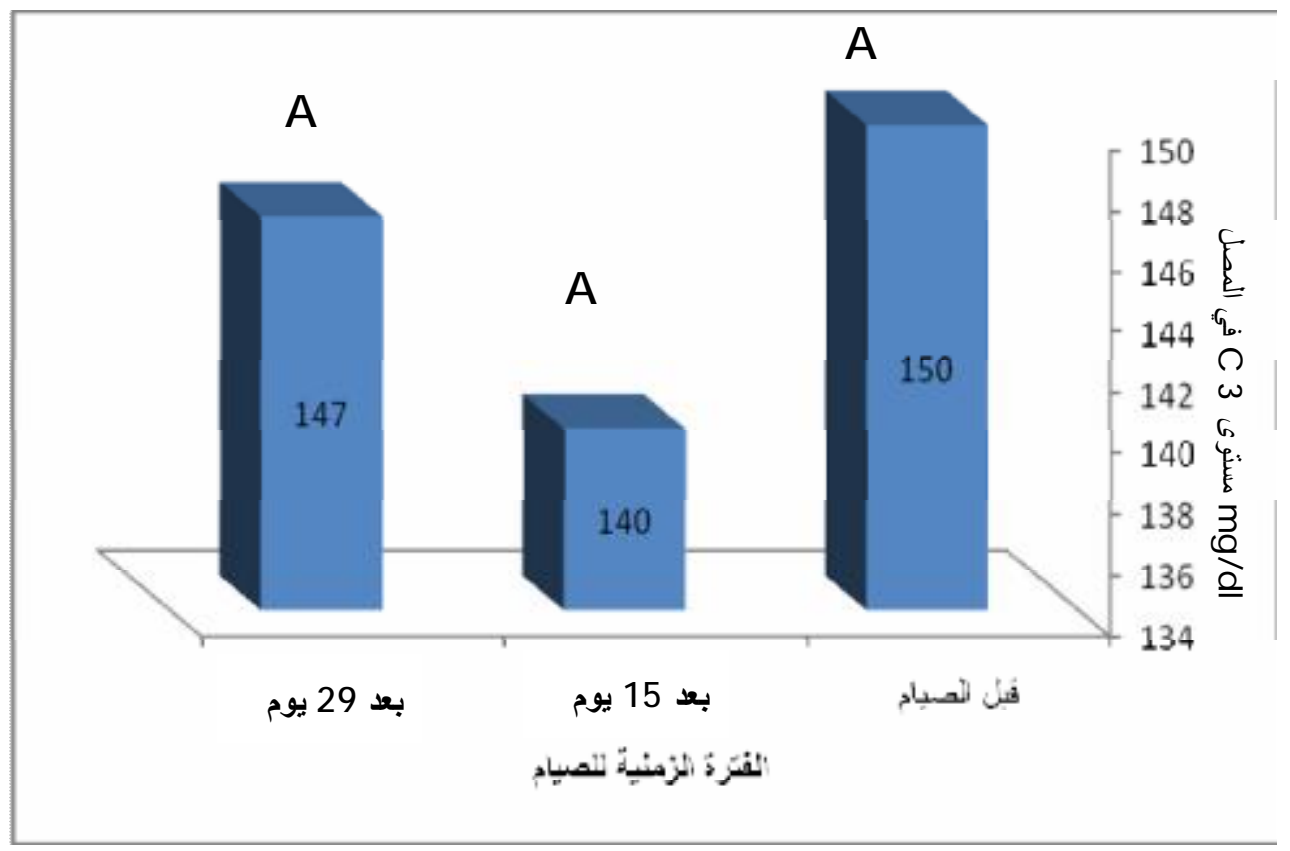

المعدلات ذات الحروف المختلفة تعني وجود فرق معنوي عند مستوى احتمالية 0.01 حسب اختبار دنكن

الثكل 4 : مستوى الجزء C3 من المتمح في مصول المتطوعين قبل و خلال صيام شهر رمضان 
زياد ذنون الرسام

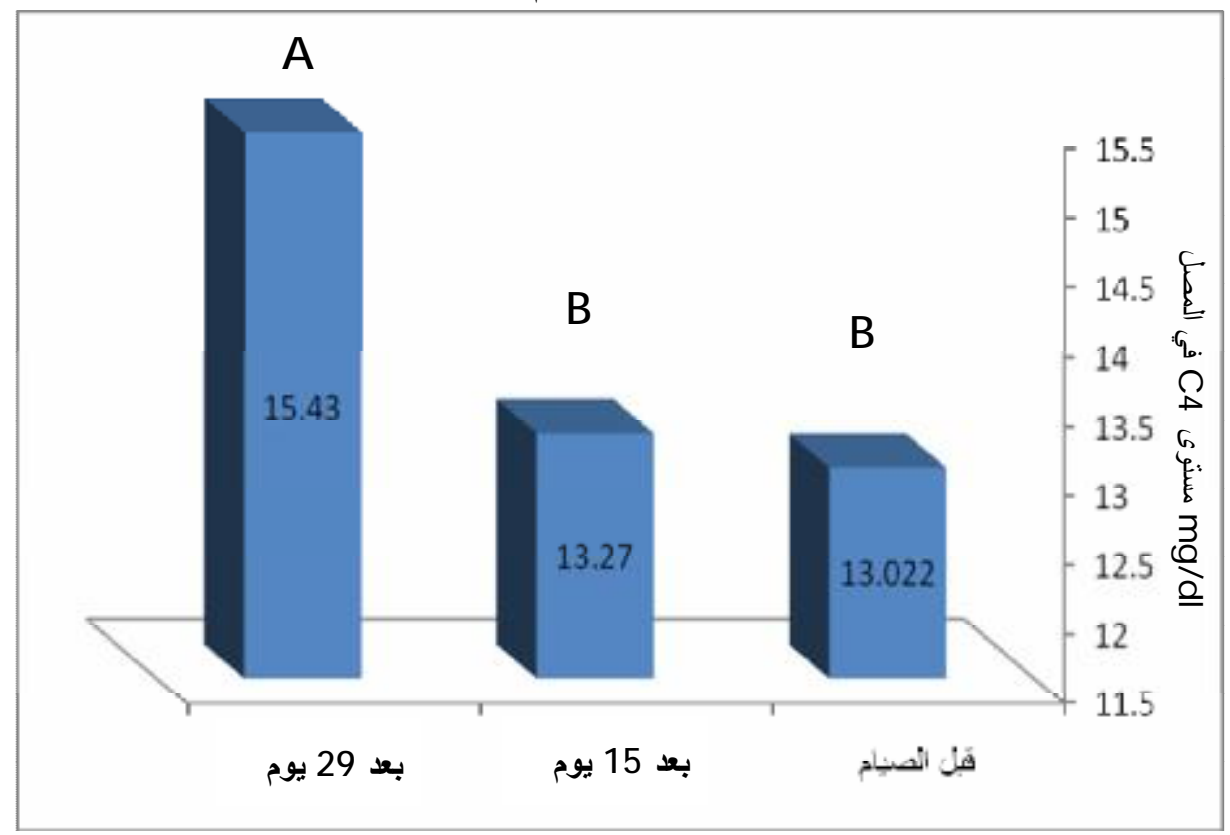

المعدلات ذات الحروف المختلفة تعني وجود فرق معنوي عند مستوى احتمالية 0.01 حسب اختبار دنكن

الشكل 5 : مستوى الجزء C4 من المتمم في مصول المتطوعين قبل و خلال صيام شهر رمضان

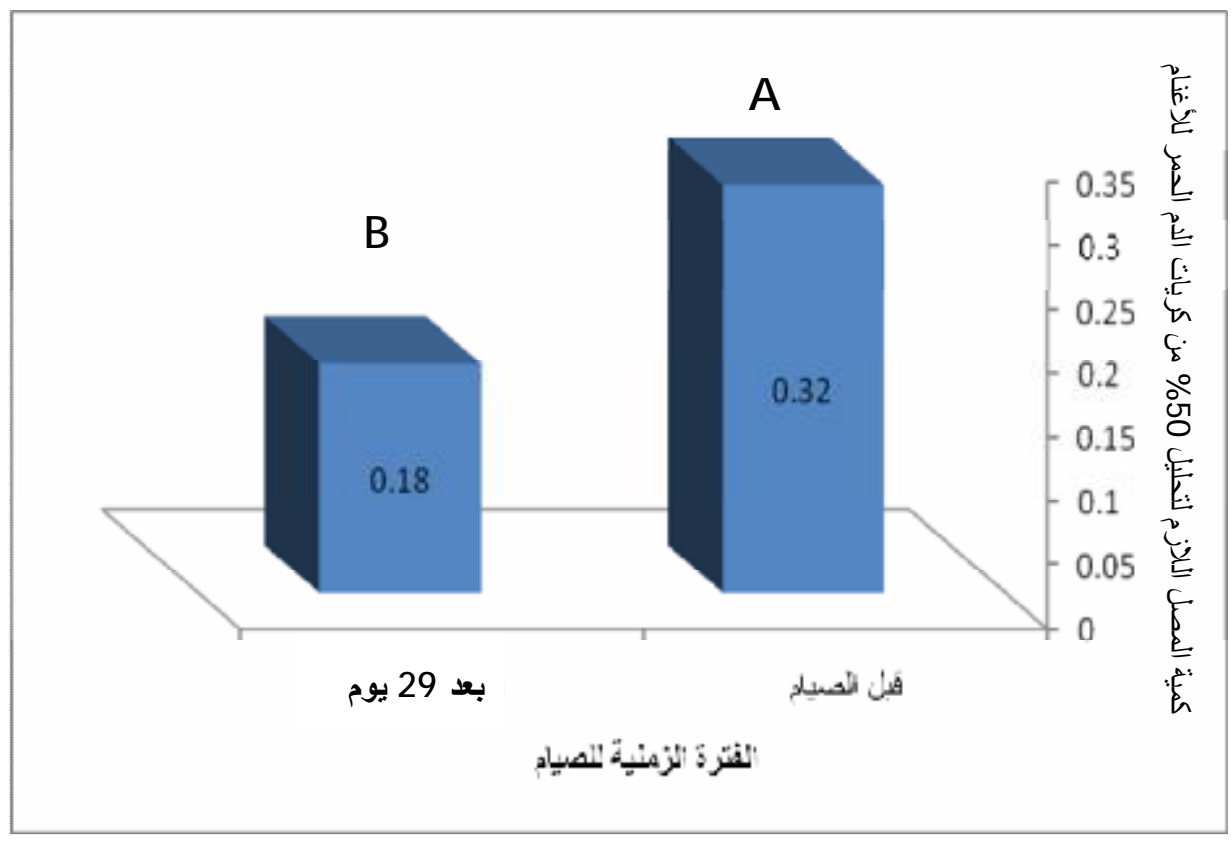

المعدلات ذات الحروف المختلفة تعني وجود فرق معنوي عند مستوى احتمالية 0.01 حسب اختبار دنكن.

الشكل 6 : كمية المصل اللازم لتحليل 50\% من كريات الدم الحمر للأغنام 


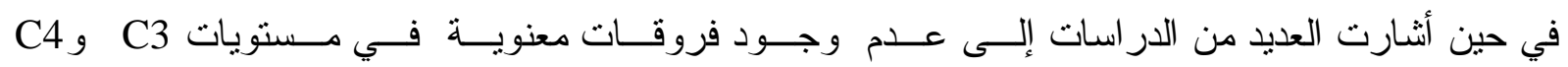

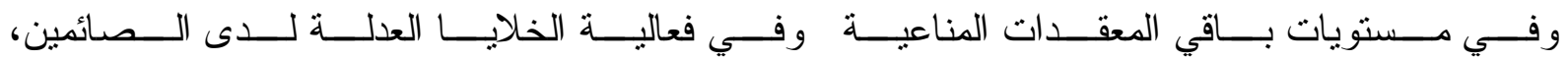

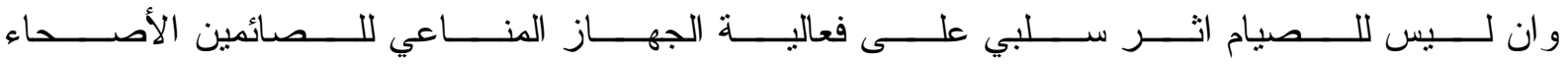
(Sakr , 1975 ; Allen and Pruitt, 1982 ; Maliji et al., 2006 ; Latifynia et al., 2008) في نتائج الدر اسات التي تناولت موضوع الصيام قد يعود إلى التتوع في الطبيعة السلوكية والمعاثية للمجتمع فضلا عن طبيعة المناخ و الفصل الذي حل فيه شهر رمضان حيث أن لدرجات الحرارة المرتفعة اثر كبير في تفاوت النتائج المسجلة. 
تثير النتائج الموضحة في الثكل (6) وجود انخفاض معنوي في كمية المصل اللازمسـة لإحـــداث

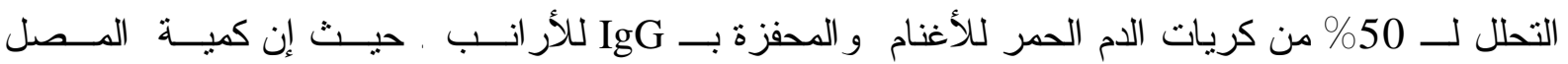
المأخوذة من الصائمين بعد 29 يوماً من الصيام كانت أكثر فعالية في تحليل كريات الــدم الحمــر للأغنــام

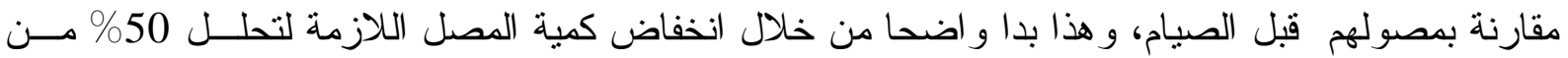
الكريات الحمر ـ إن التحري عن قدرة المتمم في تحديد خلايا الهدف واستمر ار فعاليته إلى نهاية السلسلة تقاس من خلال تحديد قدرة المصل لتحليل 50\% من كريات الدم الحمر (CH50) و التي في الغالب تتمثـل بكميــة

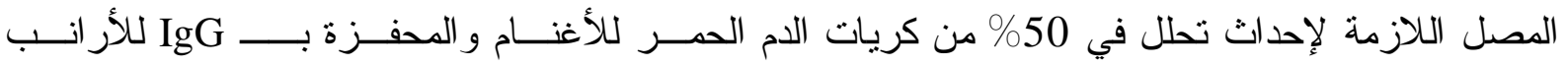
(Ahmed and Peter, 1995) يشير معدل (CH50) الطبيعي إلى إن مكونات المتمم من C1 الى C9 موجودة

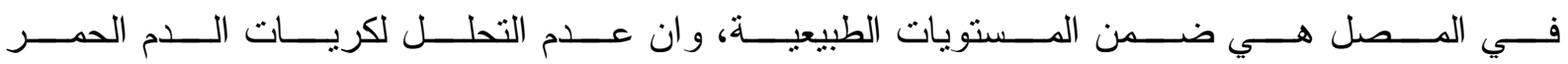
يدل على وجود نقص في و احد أو أكثر مــن بروتينــات المــتم، و ان الانخفــاض فـي فعاليــة (CH50)

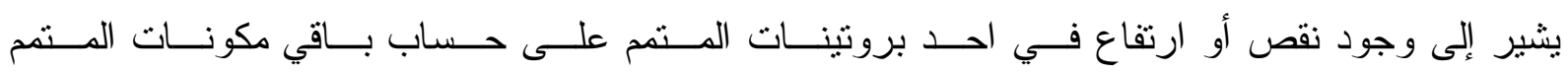
.(Hebert et al., 1991; Whaley et al., 1993 ; Jaskowski et al., 1999) أنثارت العديد من الدر اسـات إلى تـسجيل فروقـات معنويــة فــي الاســتجابة المناعيـة خــلال الــصيام (Allen and Pruitt, 1982 ; Gustaviani et al., 2004)

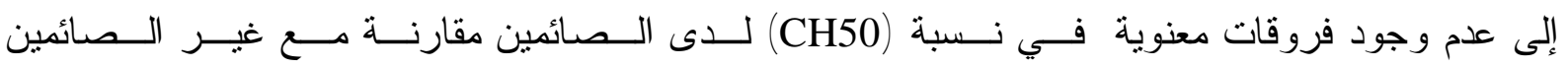

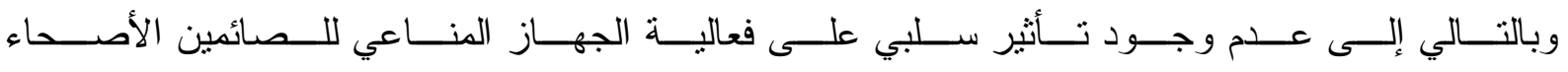
(Maliji et al., 2006 ; Latifynia et al., 2007 ; Latifynia et al., 2008)

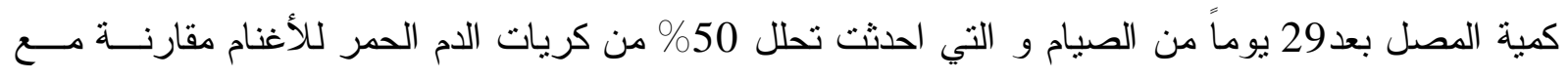
كميته قبل الصيام، إلى زيادة بروتينات المصل بسبب حالة الجفاف الناتجة عن فقد الـسو ائل خــلال الــصيام . (Gutiérrez et al., 2001 ; Azizi ,2002) أوضحت الدر اسة وجود علاقة عكسية بين كمية المصل اللازمة لإحداث التحلل لــ 50\% من كريات الدم للأغنام (CH50)، ومستوى كل من عنصر المغنيسيوم و الجز \& C4 من المتمم في مصول الصائمين، و أظهرت أيضا ارتباط مستوى الجزء C3 من المتمم بعلاقة عكسية مع مستوى عنصر الكالسيوم وبعلاقــة طردية مع مستوى عنصر البوتاسيوم في المصل، وارتبط مستوى الكالسيوم بعلاقــة عكـسية مــع مـسنتوى عنصر البوتاسيوم في المصل. إن لنقص ايونات المغنيسيوم في المصل اثر و اضـح على الاستجابة المناعيــة للجسم و غالبا ما يتر افق معه زيادة في مستوى C3 و التي زيادتها لها دور ايجـابي فـي تتـشيط الاســتجابة المناعية خلال الطور الحاد، و إن النقص في فعالية المتمم لم ترنبط بالنقص الحاصل في ايونات المغنيـسيوم في مصول الحيو انات المختبرية (Bussière et al., 2003). ومن ما تقدم يتضح لنا أن ليس للصبام أي نأثير سلبي على فعالية الجهاز المناعي لجسم الأصحاء بل على العكس من ذلك فان الصبام قد يتـسبب بإحــداث 


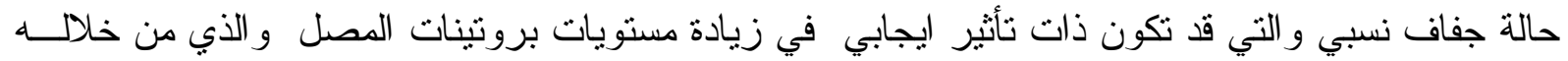
سوف تضفي شيء من الايجابية على صحة الإنسان السليم. وخير ما نختم به هو كلام الله عز وجل : ((شَهْرُ

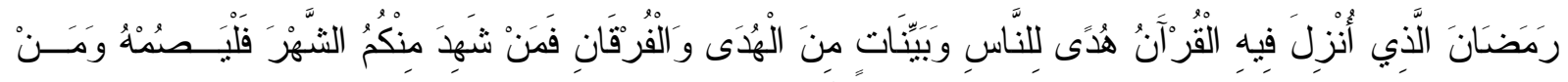

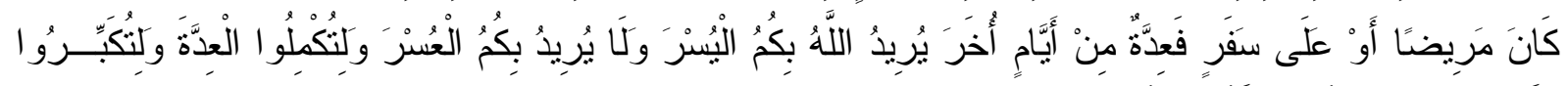

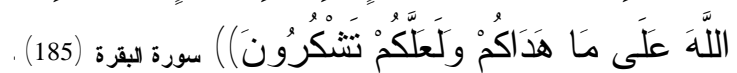

\section{المصادر الأجنبية}

Abolfazl, K. ; Mostafa, N. ; Soleiman, K. ; Jafar, N. ; Hosein, Y. ; Amirgholi, J. (2007). Impact of maternal Ramadan fasting on growth parameters in exclusively breast-fed Infants. Iran J. Pediatr, 17 (4), 345-352.

Ahmed, AE.E. ; Peter, JB. (1995). Clinical utility of complement assessment. Clin. Diagn. Lab. Immunol. 2, 509-17.

Akuchekian, S.; Ebrahimi, A. ; Alvandian, S. (2004). Effect of the holy month of Ramadan on coping strategies. J. Rese. Med. Sci., 9 (2), 65-68.

Al-Dubeikil, K. Y. ; Abdul-Lateef, W. K. (2003). Ramadan fasting and rheumatoid arthritis. J. Bahrain Med Bulletin, 25 (2), 1-7.

Allen, R.C. ; Pruitt, B.A. (1982). Humoral-phagocyte axis of immune defense in burn patients. Chem. Oluminigenic probing. Arhc. Surg. 117, 133-40

Aylin, Y. ; Bumin, D. ' Murat, A.; Ramazan, A. ; Alpay, H . (2004). The effect of fasting month of Ramadan on the abdominal fat distribution: assessment by computed tomography. Tohoku J. of Experimen. Med. 204 (3),179-187.

Azizi, F. (2002). Research in islamic fasting and health. Ann. Saudi. Med., 22, 186-91.

Bussiere, F. I. ; Tridon, A.; Zimowska, W.; Mazur, A. ; Rayssiguier, Y. (2003). Increase in complement component $\mathrm{C} 3$ is an early response to experimental magnesium deficiency in rats. Life Sci. 73 (4) , 499-507.

Cheah, S. H. ; Chng, S. L. ; Husain, R. ; Duncan, M.T. (1990). Effects of fasting during Ramadan on urinary excretion in Malaysian muslims. British J. nutrition. 63 (2), 329-37.

Davoodabadi, A. ; Akbari, H. ; Rasoulinejad, A. (2005). The impact of fasting during the holy month of Ramadan on incidence of acute appendicitis. I.J.MS. 30 (1), 21-23.

Dikensoy, E. ; Balat, O. ; Cebesoy, B. ; Ozkur, A. ; Cicek, H. ; Can, G. (2008). Effect of fasting during Ramadan on fetal development and maternal health. J. Obste. Gynaecolo. Rese. 34 (4), 494-8.

Fedail, S. S.; Murphy, D.; Salih, S.Y.; Bolton, C.H ; Harvey, R.F. (1982). Changes in certain blood constituents during Ramadan. American J. Clinl. Nutrition., 36, 350353.

Frost, G. ; Pirani, S. (1987). Meal frequency and nutritional intake during Ramadan: a pilot study. Human Nutrition Applied Nutrition. Ann. Rev. Nutrition. 25, 237-260.

Fernandoz, F. J. ; Khan, H. L. (1971). Clinical methods for atomic absorption spectroscopy. Clin. Chemis. News. 3, 24- 29. 


$$
\text { زياد ذنون الرسام }
$$

Gustaviani, R. ; Soewondo, P. ; Semiardji, G. ; Sudoyo, A.W. (2004). The influence of calorie restriction during the Ramadan fast on serum fructosamine and the formation of beta hydroxybutirate in type 2 diabetes mellitus patients. Acta. Medica. Indonesiana. 36(3),136-141 .

Gutierrez, A.; Gonzalez-Gross, M.; Delgado, M. ; Castillo, M J. (2001). Three days fast in sportsmen decreases physical work capacity but not strength or perception-reaction time. International J. sport nutrition and exercise metabolism. 11 (4), 420-9.

Hebert, L.A.; Cosio, F.G. ; Neff, J. C. (1991). Diagnostic significance of hypo complementemia. Kidney Int. 39, 811-21.

Hudson, L. and Hay, F.C. (1980). "Practical Immunology", 2nd edn., Blackweel Scientific Publication, Oxford London. pp.142-145

Jamshid, A. (2006). Typhoid fever complicated by leukocytoclastic vasculitis. Med J. Islamic World Acad. Sci. 16 (2), 93-94.

Jaskowski, T. D.; Martins, T. B.; Litwin, C. M. ; Hill, H. R. (1999). Comparison of three different methods for measuring classical pathway complement activity. Clin. Diagn. Lab. Immunol. 6 (1), 137-139.

Kiziltan, G. ; Karabudak, E. ; Tuncay, G.; Avsar, F. ; Tuncay, P.; Mungan, O. ; Meral, P. (2005). Dietary intake and nutritional status of Turkish pregnant women during Ramadan. Saudi med. J., 26 (11), 1782-7.

Latifynia, A.; Vojgani, M.; Gharagozlou, M. J. ; Sharifian, R. (2008). Effect of Ramadan on neutrophil's respiratory burst (innate immunity) and circulating immune complex. Iran. J. Ayub Med. Coll Abbottabad,. 20(3), 128-131.

Latifynia, A ; Vojgani, M ; Abofazeli, T ; Jafarieh, H. (2007). Circulating immune complex during Ramadan. J. Ayub Med. College. 19 (2), 15-8.

Leiper, J.B.; Molla, A.M. ; Molla, A.M. (2003). Effects on health of fluid restriction during fasting in Ramadan. Europ J . Clin. Nutrition . 57(2) , 30-38.

Maliji, Gh.; Qujeq, D.; Mahdavi Omran, S. ; Habibi, T. (2006). Effects of Ramadan fasting on complement components activities C3,C4, and CH50, of human serum. Sci. J. Ilam Med. Univ. 14 (1), 50-54.

Mattson, M. P. (2005). Energy intake, meal frequency, and health. Ann Rev. Nutrition. (25), 237-260.

Male, D.; Brostoff, J.; Roth, D. B. ; Roitt, I. (2006). "Immunology", 7th edn., MOSBY ELSEVIER, Canada . pp. 87-104.

Neslisah, H.; Gulhan, H.; Ali, A. ; Aylinayaz, T. (2006). The effect of Ramadan on maternal nutrition and composition of breast milk. Pediatrics International. 48(3), 278-283.

Ramadan, J.; Mousa, M. ; Telahoun, G. (1995). Effect of Ramadan fasting on physical performance, blood and body composition. International J. Kuwait Univ. Health Sci. Centre. 4 (4), 204-212.

Ramadan, J.; Telahoun, G.; Al-Zaid, N. S. ; Barac-Nieto, M. (1999). Responses to exercise, fluid, and energy balances during Ramadan in sedentary and active males. Nutrition, 15, 735-739.

Sakr, A. H. (1975). Fasting in Islam. J. Am. Diet. Assoc. 67, 17-21.

Salim, S.; Farooq, N.; Priyamvada, S.; Asghar, M.; Khundmiri, S. J.; Khan, S.; Khan, F. ; Yusufi, A.N. (2007). Influence of Ramadan-type fasting on carbohydrate metabolism, brush border membrane enzymes and phosphate transport in rat kidney used as a model. The British J. Nutr. 98 (5), 984-90. 
Schmahl, F.W. ; Metzler, B. (1991). The health risks of occupational stress in islamic industrial workers during the Ramadan fasting period. Polish J. Occupational Med. 4, 3219-28.

Seppanen, M.; Suvilehto, J.; Lokki, M-L. ; Notkola, I-L.; Järvinen, A.; Jarva, H.; Seppälä,. I.; Tahkokallio, O.; Malmberg, H.; Meri, S. ; Valtonen, V. (2006). Immunoglobulins and complement factor $\mathrm{C} 4$ in adult rhinosinusitis. Clin. Exp. Immunol., 145(2), 219-227

Snyderman, R. ; Pike, M.C. (1975). Interaction of complex polysaccharides with the complement system: effect of calcium depletion on terminal component consumption. Infect Immun. 11(2), 273-279.

Spacek , J.; Jilek , P.; Buchta, V.; Főrstl , M.; Hronek , M. ; Holeckova , M. (2005). The serum levels of calcium, magnesium, iron and zinc in patients with recurrent vulvovaginal candidosis during attack, remission and in healthy controls. Mycoses. 48 (6) 391 - 395.

Tavakkoli, H.; Haghdani, S.; Emami, M.H.; Haideh Adilipour, A.; Tavakkoli, M.; Tavakkoli, M.O.(2008). Ramadan fasting and inflammatory bowel disease. Indian J. Gastroenterol. 27 (6), 239-241.

Whaley, K. Loos, M. ; Weiler, J. M. (1993). Complement in health and disease. Boston Kluwer. Acad. Publish, 1-32. 